\title{
The CFTR Ion Channel: Gating, Regulation, and Anion Permeation
}

\author{
Tzyh-Chang Hwang ${ }^{1}$ and Kevin L. Kirk ${ }^{2}$ \\ ${ }^{1}$ Dalton Cardiovascular Research Center, University of Missouri-Columbia, Columbia, Missouri 65211 \\ ${ }^{2}$ Department of Cell, Developmental and Integrative Biology and Gregory Fleming James Cystic Fibrosis \\ Research Center, University of Alabama at Birmingham, Birmingham, Alabama 35294 \\ Correspondence: klkirk@uab.edu
}

Cystic fibrosis transmembrane conductance regulator (CFTR) is an ATP-gated anion channel with two remarkable distinctions. First, it is the only ATP-binding cassette (ABC) transporter that is known to be an ion channel-almost all others function as transport ATPases. Second, CFTR is the only ligand-gated channel that consumes its ligand (ATP) during the gating cycle-a consequence of its enzymatic activity as an $A B C$ transporter. We discuss these special properties of CFTR in the context of its evolutionary history as an $A B C$ transporter. Other topics include the mechanisms by which CFTR gating is regulated by phosphorylation of its unique regulatory domain and our current view of the CFTR permeation pathway (or pore). Understanding these basic operating principles of the CFTR channel is central to defining the mechanisms of action of prospective cystic fibrosis drugs and to the development of new, rational treatment strategies.

B eing a member of the ABC (ATP-binding cassette) protein superfamily (Riordan et al. 1989), Cystic fibrosis transmembrane conductance regulator (CFTR) shows the characteristic topology of two transmembrane domains (TMDs), each conjoined to a cytoplasmic nucleotide-binding domain (NBD1 and NBD2). Unique to CFTR, however, is a regulatory $(\mathrm{R})$ domain connecting the two pseudo-symmetrical halves, each composed of a TMD and an NBD (Fig. 1A). With very few exceptions, members of the ABC transporter family harvest the energy of ATP hydrolysis to drive an uphill movement of a wide variety of substrates into (importers) or out of (exporters) the cell. ABC importers, present exclusively in prokaryotes, are important for up- take of nutrients (Rees et al. 2009). ABC exporters, on the contrary, are found in all kingdoms of life. In humans, phytogenetic analysis classifies $48 \mathrm{ABC}$ proteins into seven subfamilies (ABCA-ABCG) and CFTR belongs to the ABCC subfamily (Dean and Annilo 2005).

While other members of the ABCC subfamily use the free energy of ATP hydrolysis to export large hydrophobic anions (Leier et al. 1994; Loe et al. 1996), CFTR is a bona fide ATP-gated ion channel (Anderson et al. 1991a; Nagel et al. 1992), carrying out the function of passive diffusion of chloride ions (Bear et al. 1992). However, phosphorylation of the $\mathrm{R}$ domain by the cAMP-dependent protein kinase (PKA) is a prerequisite for channel gating by ATP (see

Editors: John R. Riordan, Richard C. Boucher, and Paul M. Quinton

Additional Perspectives on Cystic Fibrosis available at www.perspectivesinmedicine.org

Copyright (C) 2013 Cold Spring Harbor Laboratory Press; all rights reserved; doi: 10.1101/cshperspect.a009498

Cite this article as Cold Spring Harb Perspect Med 2013;3:a009498 
T.-C. Hwang and K.L. Kirk

A

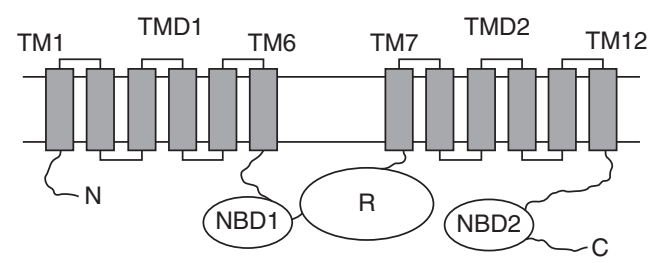

B

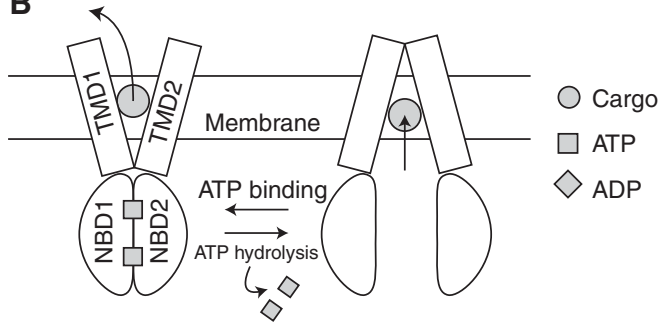

Figure 1. CFTR topology and alternating access model for ABC transporters. (A) Simplified topological model emphasizing the domain structure of a CFTR chloride channel, including cytoplasmic amino $(\mathrm{N})$ and carboxyl (C) termini, two nucleotide-binding domains (NBD1, NBD2), the regulatory (R) domain, and the predicted transmembrane domains (TMD1 and TMD2). (B) Alternating access model for the ABC transporter that extrudes its substrate (i.e., exporter). In the apo state of exporters, the configuration of the TMDs is inward facing. The two NBDs are far apart. Once ATP binds to the Walker A and B motifs of the "head" subdomain, two NBDs dimerize in a head-to-tail configuration and this NBD dimerization provides the driving force to flip-flop TMDs so that the substrate binding site is now outward facing.

below for details). Crystallographic studies of several $A B C$ proteins reveal at least two conformations of the protein: "inward-facing" TMDs with monomeric ATP-free NBDs and "outward-facing" TMDs with dimeric ATP-bound NBDs (Rees et al. 2009). A popular model has since been proposed (Fig. 1B). For ABC exporters, in the absence of ATP, the substrate-binding site in TMDs is accessible from the cytoplasmic side of the membrane; ATP binding triggers dimerization of the two NBDs, which is accompanied by conformational changes of TMDs so that the substrate-binding site now faces the extracellular environment.

Although the cargos for CFTR and ABC exporters differ immensely, a few pieces of evidence support the close evolutionary relationship between CFTR and ABC exporters. First, their TMDs conform to a " $6+6$ " topological fold (Fig. 1A). Second, crystallographic studies of both prokaryotic and eukaryotic ABC exporters have revealed that TM6 and TM12 line the substrate translocation pathway (Dawson and Locher 2006; Ward et al. 2007; Aller et al. 2009). Indeed, numerous experimental data have indicated that both TM6 and TM12 form part of the chloride permeation pathway in the CFTR channel (see below). Third, CFTR's two NBDs show canonical architectures including Walker A and $B$ sequences and an $A B C$ signature motif conserved among all $\mathrm{ABC}$ proteins (Lewis et al.
2004, 2005) and, just like other ABC proteins, ATP binding to CFTR's NBDs induces dimerization of the two NBDs in a head-to-tail configuration (Mense et al. 2006). Finally, a wealth of data in the literature have indicated the presence of an anion-binding site in the CFTR pore that can accommodate large hydrophobic anionic channel blockers as if this apparent imperfect design of the pore represents an evolutionary vestige of a primordial $\mathrm{ABC}$ exporter evolving into an ion channel (Chen and Hwang 2008).

Based on the ATP-bound configuration of $A B C$ exporters, several homology models of CFTR's "open state" were composed (Serohijos et al. 2008; Alexander et al. 2009; Mornon et al. 2009; Norimatsu et al. 2012). However, these homology models, like their counterparts of $\mathrm{ABC}$ exporters, all lack an uninterrupted pathway for ion permeation. Although these structural models make sense for an active transporter, which prohibits simultaneous exposure of the substrate translocation pathway to both sides of the membrane, a lack of continuous diffusive pathway for a presumed "open" channel conformation is troublesome for an ion channel. Several articles published in recent years have proposed that the gate of CFTR is located at the extracellular end of the pore; whereas the gate at the cytoplasmic end is degraded so that a transporter protein can metamorphose into an ion channel (Gadsby et al. 2006; Chen and Hwang 
2008; Jordan et al. 2008). This proposition is supported by a very recent report providing strong evidence that the gate of CFTR is indeed located more to the extracellular part of the pore (Bai et al. 2011; but cf. El Hiani and Linsdell 2010; Wang and Linsdell 2012).

\section{GATING OF CFTR CHANNELS BY ATP BINDING AND HYDROLYSIS}

CFTR channels typically open when ATP docks in the ATP-binding site on each NBD. Unlike classical ligand-gated ion channels (e.g., the nicotinic acetylcholine receptor), CFTR's ligand ATP is consumed during the gating cycle to mediate channel closure. This phenomenon, unique to CFTR, likely reflects the evolutionary relationship of CFTR; an ABC transporter turned an ion channel (Gadsby et al. 2006; Chen and Hwang 2008). It is also interesting that, although NBD2, with all the conserved motifs, is capable of catalyzing ATP hydrolysis, $\mathrm{NBD} 1$, in contrast, is degenerated in the head subdomain with several substitutions that abolish its ATPase activity (Aleksandrov et al. 2002; Basso et al. 2003; Stratford et al. 2007). Because $\mathrm{Mg}^{2+}$ is an essential cofactor for enzyme-catalyzed ATP hydrolysis, this evolutionary relationship also provides an explanation for why it is MgATP, not ATP, which gates CFTR effectively (Dousmanis et al. 2002). $\mathrm{Mg}^{2+}$ not only helps in ATP binding by interacting with the Walker B aspartate, but is also essential for ATP hydrolysis that plays a major role in closing the channel (Gunderson and Kopito 1994; Hwang et al. 1994; Ramjeesingh et al.1999; Zeltwanger et al. 1999; Vergani et al. 2005; Stratford et al. 2007).

The strategies Mother Nature employs to stabilize ATP in the ATP-binding site of an NBD monomer were revealed by crystallographic studies of NBDs from $\mathrm{ABC}$ proteins, including those of CFTR (Lewis et al. 2004, 2005). First, upstream of the Walker A motif, the side chain of an aromatic amino acid (CFTR: NBD1, tryptophan 401 or W401; NBD2, tyrosine 1219 or Y1219) forms a $\pi$-electron stacking interaction with the adenine ring of ATP. Second, the positive charge of the Walker A lysine (CFTR: NBD1, K464; NBD2, K1250) forms an electrostatic in- teraction with the negative charge of the $\gamma$-phosphate. Third, the side chain of the Walker B aspartate (D572 in NBD1 and D1370 in NBD2 of CFTR) coordinates the $\mathrm{Mg}^{2+}$ ion. These structural studies of $A B C$ proteins in the past decade (Hung et al. 1998; Diederichs et al. 2000; Gaudet and Wiley 2001; Karpowich et al. 2001; Yuan et al. 2001; Smith et al. 2002; Chen et al. 2003; Schmitt et al. 2003; Verdon et al. 2003; Lewis et al. 2004, 2005) have provided rich molecular details that can be applied to facilitate functional exploration of CFTR. For example, Zhou et al. (2006) used site-directed mutagenesis and high-resolution single-channel recording to investigate the contribution of the aromatic residues W401 in NBD1 and Y1219 in NBD2 that interact with the adenine ring of ATP. Their data suggest that the interaction of ATP with NBD2 is critical for channel opening by ATP, whereas the interaction of ATP with NBD1 is less important for channel opening (Zhou et al. 2006). Similar functional asymmetry of CFTR's two NBDs was observed when the Walker A lysine residues in NBD1 (K464) and 2 (K1250) were mutated (Powe et al. 2002; Vergani et al. 2003). The critical role of this $\pi$-electron stacking interaction between ATP and the binding pocket also explains why pyrophosphate (PPi), a ligand without the adenine ring for this stacking interaction, shows a very low apparent affinity as a channel opener (Tsai et al. 2009). Lately, capitalizing on both structural information and bioinformatics, Szollosi et al. (2010) were able to experimentally show an induced fit mechanism following ATP binding to the head subdomain of NBD2 during CFTR gating.

For channel closing, it has long been proposed that hydrolysis of ATP is a prerequisite for fast channel closure as nonhydrolyzable ATP analogs such as AMP-PNP or pyrophosphate (PPi) lock the CFTR $\mathrm{Cl}^{-}$channel in a stable open state for tens of seconds in the presence of ATP (Gunderson and Kopito 1994; Hwang et al. 1994). Moreover, this locked-open phenotype is also observed with ATP as a ligand when hydrolysis is abolished by mutations at residue K1250 or glutamate 1371 (E1371), both essential for effective ATP hydrolysis (Gunderson and Kopito 1995; Ramjeesingh et al. 1999; Zeltwanger 
et al. 1999; Powe et al. 2002; Bompadre et al. 2005b; Vergani et al. 2005; Stratford et al. 2007). But is hydrolysis per se closing the gate as suggested by a thermodynamic study of CFTR gating (Csanády et al. 2006)? Or is it the release of the hydrolytic products that is associated with gate closure (Wang et al. 2009)? These questions remain to be answered.

\section{Roles of NBD Dimerization in CFTR Gating}

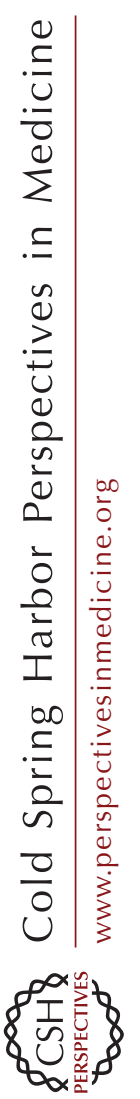

Perhaps the most intriguing mystery about CFTR gating is how the channel uses ATP binding and hydrolysis to drive conformational changes. Using mutant cycle analysis, Vergani and her colleagues provided convincing evidence supporting the idea that ATP binding induces the formation of an NBD dimer, which coincides with gate opening (Vergani et al. 2005). Cross-linking studies also showed that CFTR's two NBDs may form a canonical dimer seen in other members of the ABC proteins (Mense et al.2006). If dimerized NBDs represent the open channel configuration, it seems natural to contemplate the idea that separation of the dimer closes the gate (Vergani et al. 2005), based on the analogous structural information from other ABC proteins (Fig. 1B). Two recent studies provide experimental evidence contradicting this popular theory.

Using nonhydrolyzable ATP analogs (e.g., PPi or AMP-PNP) as ligands, Tsai et al. (2009) showed that contrary to ATP-gated channels that show an open time of $\sim 400 \mathrm{~ms}$ (Zeltwanger et al. 1999), the open time for PPi-opened channels is $\sim 30 \mathrm{~s}$ (inferred from deactivation rates) when this ligand is applied shortly after removal of ATP, but the open time becomes much shorter $(\sim 1.5 \mathrm{~s})$ if PPi is applied minutes after ATP washout (Tsai et al. 2009). Thus, CFTR channels, once primed by ATP, respond to PPi differently depending on the washout time. The two open states distinguished by their respective lifetime of $1.5 \mathrm{~s}$ and $30 \mathrm{~s}$ demand the presence of two closed states $C_{1}$ and $C_{2}$, from which PPi exerts its effects (Fig. 2).

As the stability of this $\mathrm{C}_{2}$ closed state is decreased by mutations at the composite site 1 (formed by the head subdomain of NBD1 and the tail subdomain of NBD2), but increased by
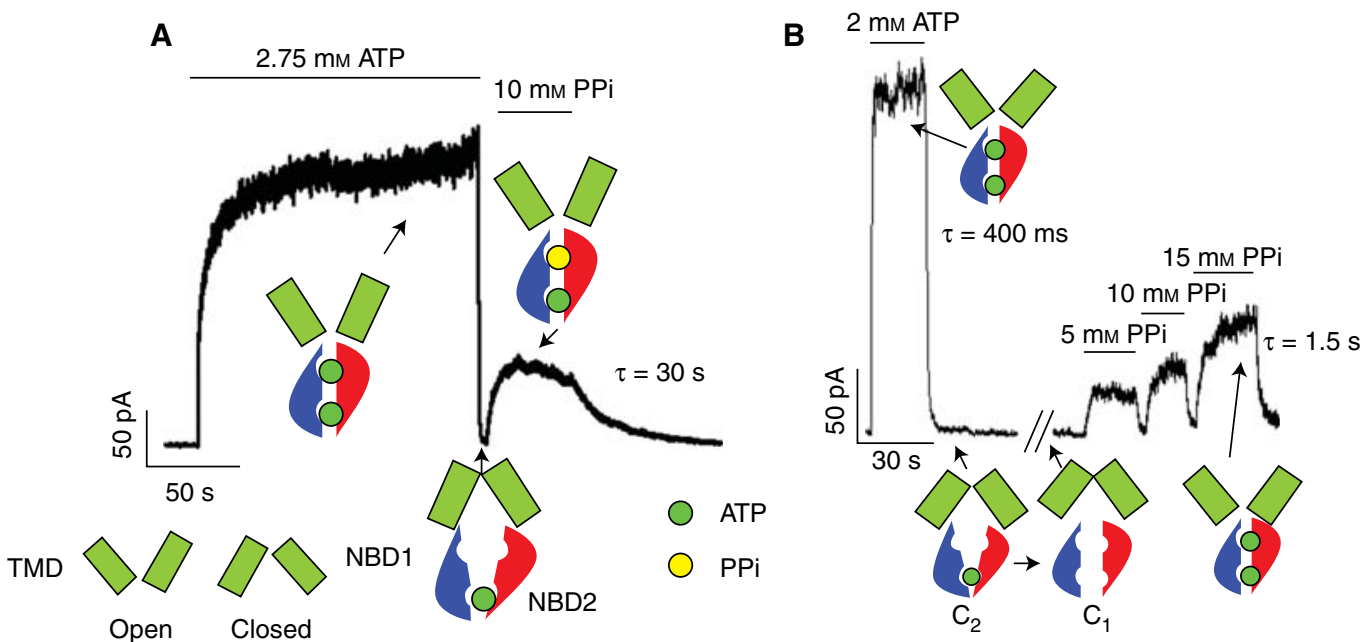

Figure 2. State-dependent modulation of CFTR gating by PPi. (A) A continuous macroscopic recording of WTCFTR in an inside-out patch showing a slow response to PPi $10 \mathrm{~s}$ after removal of ATP. However, once the channels were opened by PPi, they closed very slowly with a relaxation time constant $(\tau)$ of $\sim 30 \mathrm{~s}$. (B) Three minutes after ATP washout, CFTR channels responded to PPi in a dose-dependent manner, but with a much shorter open time $(\tau=1.5 \mathrm{~s})$. However, robust response to PPi can be restored by ATP priming again (not shown). Cartoons depict proposed configurations of NBDs. (Modified from Tsai et al. 2009; reprinted, with permission, from the author.) 
priming the channel with a high-affinity ATP analog, $\mathrm{N}^{6}$-phenylethyl-ATP (or P-ATP), it was proposed that the $\mathrm{C}_{2}$ closed state contains a partial NBD dimer with the composite site 1 remaining connected by the bound ATP, whereas the composite site 2 is separated after ATP is hydrolyzed. This hypothesis predicts that if one would replace both ATP molecules bound at the NBD dimer interface, two distinct time courses of ligand substitution should occur. Indeed, in a follow-up study, Tsai et al. (2010) showed that on switching the ligand from ATP to P-ATP, a ligand with a higher potency as well as an increased efficacy compared to ATP (Zhou et al. $2005)$, open probability $\left(P_{\mathrm{o}}\right)$ increases in two steps: an immediate increase of the $P_{\mathrm{o}}$ with an increase of the opening rate, and a $\sim 30$-s delayed increase of the $P_{\mathrm{o}}$ with an increased open time (Tsai et al. 2010). Thus, ATP remains sandwiched between the head of NBD1 and the tail of NBD2 for tens of seconds while the channel has undergone several rounds of the opening/ closing gating cycle.

These studies lead to a new model for the coupling mechanism between NBD motions and opening/closing of the gate in TMDs (Fig. 3). This model envisages "limited motions" of the NBD dimer in the completion of one catalysis (and gating) cycle. Of note, this model is supported by a report that employed a completely independent approach (Szollosi et al. 2011). The significance of this new model lies in the fact that all members of the ABCC subfamily possess only one catalysis-competent site in their NBDs. Thus, if these limited motions were shared features of all members of this subfamily, one would have to ask if a complete disengagement of the NBDs is required for all other ABC exporters that hold two catalysiscompetent sites (Fig. 1B).

\section{Future Perspectives Regarding ATP-Dependent Channel Gating}

The discussion above mainly focuses on gating of CFTR by ATP-induced NBD dimerization and hydrolysis-triggered partial or complete separation of the NBD dimer. The model in Figure 3, derived from years of biochemical and biophys-

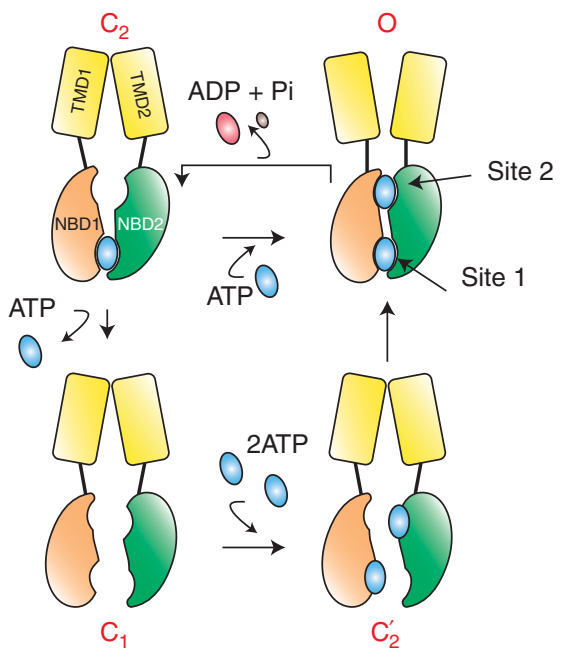

Figure 3. A simplified model for CFTR gating. The diagram illustrates dominant gating transitions $\left(\mathrm{C}_{2}\right.$ $\leftrightarrow \mathrm{O})$ and a second gating cycle $\left(\mathrm{C}_{1} \rightarrow \mathrm{C}_{2}{ }^{\prime} \rightarrow \mathrm{O} \rightarrow\right.$ $\mathrm{C}_{2} \rightarrow \mathrm{C}_{1}$ ) involving complete disengagement of the two NBDs. It should be noted that the transition from $\mathrm{O}$ to $\mathrm{C}_{2}$ likely contains many transitional states that await identification. The model also implicates a closed state wherein the two NBDs are separated with only one ATP molecule bound to NBD1. (Adapted from Tsai et al. 2010; reprinted, with permission, from the author.)

ical studies of CFTR gating, is still an oversimplification. For example, there must be several other states existing when the channel is on its way to closure (i.e., $\mathrm{O} \rightarrow \mathrm{C}_{2}$ ) because ATP will be hydrolyzed first followed by opening of the dimer interface for the dissociation of hydrolytic products, $\mathrm{ADP}$ and $\mathrm{Pi}$. As the $\mathrm{O} \rightarrow \mathrm{C}_{2}$ transition proceeds at a fairly fast rate and hence all those putative states are presumably very unstable, capturing these states with any means will be a challenge. Nevertheless, an early report by Gunderson and Kopito (1995) may shed some light on how to pursue in this direction. By scrutinizing their single-channel data, they were able to discern two conductance levels and an uneven distributions of transitions between these two open channel levels, indicating a violation of microscopic reversibility and hence a necessity of an input of free energy - in this case, from ATP hydrolysis (Gunderson and Kopito 1995). This idea of an infusion of free energy in driving 
gating transitions of CFTR is further supported by a recent detailed analysis of single-channel open time distributions (Csanády et al. 2010). These investigators showed a paucity of shortlived events that results in an unusual double exponential distributions with a negative component, indicative of a violation of microscopic reversibility. It is interesting to note that in these two pioneering investigations the data imply a strict coupling between the ATP hydrolysis cycle and the gating cycle. A one-to-one stoichiometry between the hydrolysis of one ATP molecule and one opening-closing transition fits nicely with the gating motion proposed for $\mathrm{ABC}$ transporters: one TMD-NBD complex moves synchronously on ATP-induced NBD dimerization and hydrolysis-triggered separation of the NBD dimer (Ward et al. 2007; Khare et al. 2009).

However, the concept of an obligatory coupling of NBD and TMD motions is not easily reconciled with other experimental data. In particular, it is known that CFTR channels can open, albeit with a very low frequency, in a complete absence of ATP (Bompadre et al. 2005a). This ATP-independent gating is readily seen with mutants with dysfunctional site 2 (e.g., the G551D mutation) (Bompadre et al. 2007), or interestingly constructs that lack the entire NBD2 (Cui et al. 2007; Wang et al. 2007). Furthermore, gain-of-function (GOF) mutations that enhance ATP-free CFTR gating and increase the otherwise low activities of constructs that are insensitive to ATP (G551D and NBD-deletion mutants) have been produced (Szollosi et al. 2010; Wang et al. 2010). ATP-independent gating also can be strongly enhanced by chemically modifying cysteines placed at specific locations in TM 6 (Bai et al. 2010). These results raise the question of whether NBD/TMD coupling is as tight as proposed.

The aforementioned results also blur the distinction between CFTR channel gating by ATP and the gating of a conventional ligandgated channel by reversible ligand binding and unbinding (e.g., a nicotinic acetylcholine channel). Ligand binding to the latter increases the probability of channel opening but is not absolutely required for the channel to open (Changeux and Edelstein 2005; Purohit and
Auerbach 2009). Unliganded openings are detectable and GOF mutations that increase this activity are possible. GOF mutations for conventional ligand-gated channels typically locate along the axis that links the ligand-binding site to the pore. The GOF mutations/modifications that have been discovered for CFTR locate near the NBD dimer interface (Szollosi et al. 2010), along the cytosolic loops that connect the NBDs to the TMDs (Wang et al. 2010) and within a $\mathrm{TM}$ that has been argued to line the pore (Bai et al. 2010). Thus, the CFTR gating mechanism appears to share features with the gating of conventional ligand-gated channels; notably, unliganded openings and GOF mutations along the axis that links the ligand-binding domains to the pore. Reconciling these properties of CFTR gating with the important role of ATP hydrolysis in channel closing will be essential to developing a complete picture of the CFTR gating process. It is thus safe to say that new approaches are needed to tackle this coupling mechanism that bears ramifications not only on CFTR, but also on $\mathrm{ABC}$ proteins at large. In addition, we foresee that understanding CFTR gating at a molecular level will shed light on the mechanism of action for therapeutic reagents such as Vx-770 (Kalydeco or ivacaftor), a recently FDA-approved CFTR potentiator for treating CF patients carrying the G551D mutation (Van Goor et al. 2009; Accurso et al. 2010; Ramsey et al. 2011).

\section{CFTR REGULATION BY PKA PHOSPHORYLATION}

The main stimulus of CFTR activity in vivo is its phosphorylation by cyclic nucleotide-dependent protein kinases (e.g., protein kinase A, or PKA) (Sheppard and Welsh 1999). Here we discuss the effects of PKA phosphorylation on channel gating, which is the best characterized mode of CFTR regulation by phosphorylation. Protein kinase C (Chappe et al. 2004) and AMP kinase (King et al. 2009) stimulate and inhibit CFTR activity, respectively, by less well understood mechanisms.

Unphosphorylated CFTR channels open at very low rates, if at all. Channel opening rate in an excised membrane patch is increased by 
The CFTR Ion Channel

adding PKA catalytic subunit in the presence of MgATP (Mathews et al. 1998; Wang et al. 2000). Burst duration also increases under highly phosphorylating conditions which implies that one or more PKA sites modulates channel closing (Csanády et al. 2000). Most of the important PKA sites reside within the large $\mathrm{R}$ domain between NBD1 and TM7 (Fig. 4). This approximately 150 residue domain contains eight dibasic PKA sites plus a number of monobasic sites. At least five of the PKA sites in the $\mathrm{R}$ domain (S700, S737, S768, S795, and S813) plus another site in the nearby distal portion of NBD1 (S660) are phosphorylated in vivo (Cheng et al. 1991; Hegedus et al. 2009). In general, phosphorylation of the different PKA sites has additive effects on channel activity with no one site being essential. Fifteen sites must be eliminated to create CFTR channels that are completely PKA-insensitive (Seibert et al. 1999; Hegedus et al. 2009). Two PKA sites (S737 and S768) are argued to be inhibitory on the basis of the observation that their disruption increases channel activity (Wilkinson et al. 1997; Csanády et al. 2005). These sites also appear to be phosphorylated by AMP kinase which inhibits CFTR activity possibly under hypoxic conditions (King et al. 2009).

How does the $\mathrm{R}$ domain regulate gating? The consensus view is that the unphosphorylated $\mathrm{R}$ domain inhibits channel opening, an effect that is relieved by its phosphorylation.
Rich et al. (1991) discovered that channels that lacked large portions of the $\mathrm{R}$ domain were more active in the absence of PKA stimulation. Csanády et al. (2000) subsequently showed that split CFTR molecules that lack the $\mathrm{R}$ domain and distal NBD1 (missing residues 634-836) formed channels with opening rates in the absence of PKA that approached maximal opening rates for fully phosphorylated wild-type channels. These findings confirm that channel opening is strongly inhibited by the unphosphorylated R domain (perhaps with assistance from distal NBD1). How phosphorylation relieves this inhibition is unclear but the increased negative charge at these sites on phosphorylation appears to be relevant (i.e., replacing serines in PKA sites with acidic residues creates channels that open more frequently in the absence of PKA [Rich et al. 1993]).

\section{Proposed Mechanisms of CFTR Regulation by PKA}

We consider three mechanisms: (1) R domain phosphorylation regulates NBD dimerization; (2) R domain phosphorylation affects gating independently of NBD dimerization perhaps via physical interactions among the $\mathrm{R}$ domain, the TMDs, and/or their cytosolic extensions; and (3) phosphorylation of PKA sites within NBD1 modulates NBD1-TMD interactions

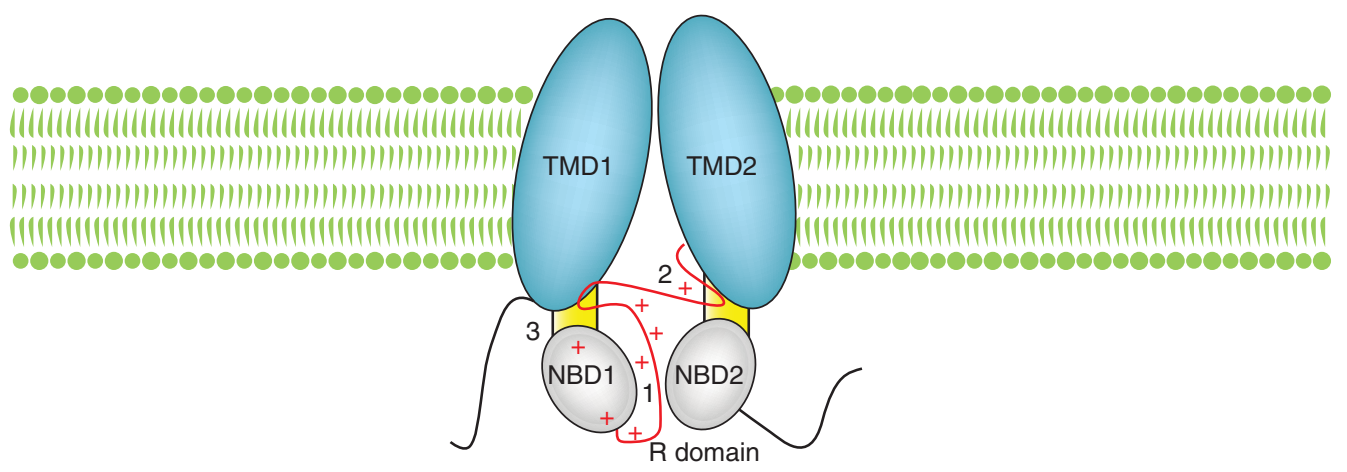

Figure 4. Three proposed mechanisms for CFTR channel regulation by PKA phosphorylation. PKA sites are indicated by the red crosses. (1) Phosphorylation of the R domain (in red) and/or distal NBD1 modulates NBD dimerization. (2) Phosphorylation more directly regulates the flexibility or packing of the TMs by direct physical interactions with the long cytosolic loops (in yellow) or with the TMs themselves. (3) Phosphorylation of sites within NBD1 stabilizes the coupling between the NBDs and the TMDs. 
(Fig. 4). These mechanisms are not mutually exclusive given the many PKA sites in CFTR.

\section{Mechanism 1}

Mense et al. (2006) showed an effect of PKA on NBD dimerization. They observed that (1) split CFTR molecules could be cross-linked by introducing cysteines at the NBD dimer interface predicted from the crystal structures of other ABC transporters, and (2) PKA activation strongly enhanced cross-linking efficiency (confirmed by $\mathrm{He}$ et al. 2008). Baker et al. (2007) provided additional support for this type of mechanism in an NMR structural analysis of an R domain fragment. This fragment bound to recombinant NBD1 in solution with most of the contact points located at or near PKA sites. PKA phosphorylation of this fragment reduced its binding to NBD1. The investigators proposed that the unphosphorylated $\mathrm{R}$ domain inhibits CFTR channel activity by binding to NBD1, which could prevent the NBD dimerization that mediates channel opening. Phosphorylation was argued to relieve this inhibition by reducing its affinity for NBD1. This is a plausible model but it does not account for all aspects of how the $\mathrm{R}$ domain controls channel gating (see below).

\section{Mechanism 2}

The $\mathrm{R}$ domain also regulates channel activity independently of ATP binding or NBD dimerization. As noted earlier CFTR truncation mutants that lack NBD2 show detectable channel activity (Cui et al. 2007).Wang et al. (2007, 2010) observed that the channel activity of an NBD2deletion mutant was strongly dependent on PKA phosphorylation but not ATP binding, an effect that required the R domain. Similarly, G551D-CFTR activity is dependent on PKA phosphorylation but not ATP binding (Bompadre et al. 2007; Wang et al. 2010). These data indicate that the $\mathrm{R}$ domain must regulate more than NBD dimerization. Conceivably, portions of the $\mathrm{R}$ domain physically interact in a PKAregulated manner with the cytosolic loops or the TMs with consequent effects on the TM confor- mational rearrangements that underlie channel gating. The apparent location of the $\mathrm{R}$ domain in electrocryomicroscopic images of purified CFTR reported by Zhang et al. (2011) is consistent with this idea. Hegedus et al. (2008) proposed a somewhat related mechanism based on the results of a computational analysis of the $\mathrm{R}$ domain. In their model, the $\mathrm{R}$ domain regulates the packing or organization of the TM helices by functioning as an "entropic spring" that controls the spacing between NBD1 and TMD2. Phosphorylation, which increases the overall size of the $\mathrm{R}$ domain in their simulations, is imagined to change this spacing with concomitant effects on TM packing.

\section{Mechanism 3}

The third mechanism is supported by the results of an NMR study by Kanelis et al. (2010). Although most PKA sites reside within the $\mathrm{R}$ domain, several sites exist within NBD1 in CFTRspecific regions termed the regulatory extension (e.g., S660) and the regulatory insertion (S422). Kanelis et al. (2010) observed dynamic interactions between the NBD1 core and these CFTRspecific regions that were inhibited by PKA phosphorylation. In addition, they detected a binding interaction between phosphorylated (but not unphosphorylated) NBD1 and a peptide that mimics a region of cytosolic loop 1 that presumably mediates the coupling between NBD1 and TMD1. The investigators concluded that NBD1-TMD interactions are dynamic and regulated by PKA phosphorylation (in this case of sites in NBD1). Such a mechanism could operate in parallel with regulation by the $\mathrm{R}$ domain to potentiate CFTR activation by PKA.

In summary, PKA phosphorylation may affect channel gating in multiple ways. Most PKA regulation is attributable to the large $\mathrm{R}$ domain with its many PKA sites. Conceivably there is a division of labor among these sites; some sites may regulate $\mathrm{NBD}$ dimerization and others may more directly regulate TM flexibility or packing via physical interactions with the TMs or their connecting cytosolic loops. But it is also possible that one predominant mechanism exists with other outcomes secondary to this primary 
mode of regulation. For example, the observed enhancement of NBD1-NBD2 cross-linking by PKA could be a secondary consequence of a more direct effect of PKA phosphorylation on TM packing. Given that the NBD dimer associates primarily with the channel open state, factors that bias the equilibrium toward the open state should also promote NBD dimerization by allosteric coupling (Kirk and Wang 2011).

\section{THE CFTR PORE}

We noted earlier that the CFTR permeation pathway likely has some similarities to the translocation pathways of other ABC transporters. But CFTR is an ion channel and not a pump, meaning that its pore must remain open to both sides of the membrane to permit anion diffusion down an electrochemical potential gradient. This property of CFTR, coupled with its preference for small anions, means that its permeation pathway cannot be inferred directly from other ABC transporters. Progress in understanding the CFTR pore has been made, however, by (1) characterizing its ion selectivity; (2) developing biophysical models of the pore based on its selectivity; and (3) using site-directed mutagenesis and chemical modification strategies to identify transmembrane helices (TMs), and residues within these TMs, that influence permeation and selectivity. Such TM residues become candidate pore-lining resides (with some caveats) and, as such, offer a first glimpse into how the pore may be structured.

\section{Anion Selectivity}

Chloride channels are less selective for different anions than most cation channels are for different cations (e.g., voltage-gated K channels) (Hille 1973). This difference reflects the necessity of a $\mathrm{K}$ channel discriminating between $\mathrm{K}$ and the equally abundant $\mathrm{Na}$. The lower selectivity of anion channels means that they can have multiple substrates that are physiologically relevant. Chloride is an important CFTR substrate in tissues that mediate salt and fluid transport such as exocrine glands (Wine and Joo 2004). CFTRmediated bicarbonate transport also is relevant in pancreatic ducts and the airways (Ishiguro et al. 2009; Kim and Steward 2009).

We must be precise when we discuss selectivity measurements. The two common measures of ion selectivity are "relative permeability" and "relative conductance." The permeability and conductance sequences of CFTR are quite different, which underscores the importance of understanding each. A CFTR permeability sequence is $\mathrm{SCN}>\mathrm{NO}_{3}>\mathrm{Br}>\mathrm{Cl}>$ $\mathrm{I}>$ acetate; the corresponding conductance sequence is $\mathrm{Cl}>\mathrm{NO}_{3}>\mathrm{Br}>$ acetate $>\mathrm{I}>$ SCN (Linsdell et al. 2000; McCarty and Zhang 2001). Relative conductance may be the more biologically relevant measure because it is an index of "throughput," i.e., the rate of flow (flux) of an ion through the pore per unit driving force. This flux is measured as a current under conditions when the test ion is the only current-carrying species. Relative permeabilities are measured from shifts in reversal potential (voltage at zero current) when a test ion is added to the side of the membrane opposite $\mathrm{Cl}$. Such reversal potential shifts typically are greatest for those ions that enter the pore most easily, not for ions with the greatest throughput. However, permeability ratios do offer insights into how anions vary with respect to their abilities to enter the pore and how they may interact with the pore walls, as discussed below.

\section{Biophysical Models of CFTR Selectivity: Dielectric Tunnels and Low-Grade Selectivity Filters}

The CFTR permeability sequence is ordered principally on the basis of the hydration energies of the permeant anions, termed a lyotropic sequence (Borman et al. 1987; Smith et al. 1999). This indicates that pore entry is dominated by the energy required to remove the ion from water. What about translation through the pore? Dawson and colleagues (Smith et al. 1999; Liu et al. 2003) showed mathematically that, on the basis of its permeability sequence and the known hydration energies of its permeant anions, the CFTR pore can be modeled as a "dielectric or polarizable tunnel" for which no specific interactions between the permeant anions 
and the pore walls need occur. The only requirement for this conceptual tunnel is that it has a dielectric constant (index of charge screening) intermediate between water (ca 80) and lipid $(<5)$; namely, about 20 for the CFTR pore. Selectivity arises because those anions that partition more effectively into the pore from water (e.g., SCN) also reside longer on average within the pore (bind more tightly) because of a greater difference between their interaction energies with water (hydration energies) and with the lower dielectric pore interior (solvation energies). To a first approximation this bias-type selectivity mechanism (Smith et al. 1999) describes how permeant anions traverse the CFTR pore with of course the limit that some anions are too big to pass through the pore in the first place (Alexander et al. 2009).

This argument does not exclude the possibility that permeant anions physically interact with pore-lining residues in ways that influence CFTR selectivity. There is good evidence for a narrowing of the CFTR pore that is lined by residues that physically interact with anions as they traverse the channel. This evidence comes from mutational and chemical modification studies of the various TMs with TM6 generating the most enthusiasm. CFTR may have a sort of low-grade selectivity filter-not on a par with the selectivity filters of voltage-gated $\mathrm{K}$ channels (Jiang et al. 2003)—but influencing anion selectivity nonetheless.

\section{Pore-Lining TMs}

TM mutagenesis experiments were first performed by Anderson et al. (1991b) to determine if the newly cloned CFTR cDNA encoded an anion channel. By mutating two lysines in predicted TMs 1 and 6 (K95 and K335) to acidic residues they altered the relative halide permeability sequence of the cAMP-activated current in CFTR-expressing cells. This was the strongest evidence at the time that CFTR is an anion channel. However, these results do not mean necessarily that $\mathrm{K} 95$ and $\mathrm{K} 335$ form part of a selectivity filter or even line the pore. A caveat in this sort of analysis is the possibility that an engineered mutation indirectly affects pore ar- chitecture. In this regard, K95 substitutions have multiple, complicated effects on permeation. K95 has been argued more recently to attract anions into the pore from the cytosol by residing near the entrance to the pore (inferred from strong voltage-dependent rectification of macroscopic currents for certain K95 mutants) (Linsdell 2006; Zhou et al. 2010). Some K95 mutations (e.g., K95S) also markedly decrease single channel conductance at both depolarizing and hyperpolarizing voltages (Zhou et al. 2010), which implies an effect on pore structure separate from a charge-attracting role for $\mathrm{K} 95$.

With the difficulty in interpreting K95/ TM1 data as a cautionary tale, we return to TM6 for which a consensus view has emerged. Linsdell et al. (2000) first reported that mutations at residues F337 and T338 in TM6 strongly affected the permeability sequence. Those findings were extended by testing additional TM6 mutants (McCarty and Zhang 2001; Ge et al. 2004) and by using cysteine scanning to explore the accessibility of TM6 residues (Alexander et al. 2009; Bai et al. 2010; El Hiani and Linsdell 2010). Alexander et al. (2009) compared the effects of two types of reactive compounds on a series of TM6 cysteine mutants: large methanethiosulfonate (MTS) compounds that are not expected to traverse the pore and smaller pseudohalides that both traverse the CFTR pore and are thiol reactive $\left(\mathrm{Ag}(\mathrm{CN})_{2}\right.$ and $\left.\mathrm{Au}(\mathrm{CN})_{2}\right)$. The impermeant MTS reagents reacted with cysteines at positions 331 to 338 but no deeper into the pore when added to the extracellular side. Conversely, the permeant pseudohalides reacted with cysteines positioned along the entire length of TM6 (to 353). These results confirmed that TM6 residues line the pore and strongly supported the proposed narrowing near position 338 from the extracellular end of the pore (Linsdell et al. 2000; McCarty and Zhang 2001). On the other hand, applying bulky MTS reagents from the cytoplasmic side of the channel identified position 341 as the accessibility limit (Bai et al. 2010; El Hiani and Linsdell 2010). Thus, it appears that the pore is constructed with two fairly accessible internal and external vestibules with a "bottleneck" that traverses only one helical turn (Norimatsu et al. 2012). 
What about the other TMs? We noted above the evidence (with caveats) for a role for TM1. TMs 5, 11, and 12 also have been argued to contribute to the pore based on mutagenesis and cysteine scanning results (Zhang et al. 2000a,b; Fatehi and Linsdell 2009). Recent results from systematic cysteine scanning studies not only suggest that TM12 assumes an $\alpha$-helical secondary structure, but also support the idea that TM12 is part of the pore-forming domain (Bai et al. 2011; cf. Qian et al. 2011). This latest study also provides new evidence supporting the evolutionary relationship between the CFTR channel and ABC exporters. First, the pattern of state-dependent accessibility for MTS reagents suggests that, during gating transitions, TM12 undergoes a rotational movement, which has long proposed to be part of the molecular motions for ABC transporters (Rosenberg et al. 2001; Gutmann et al. 2010). Second, using MTS reagents with different sizes, Bai et al. (2011) showed that the opening of CFTR's gate is associated with a narrowing of the cytoplasmic entrance of the pore - a result that makes sense if CFTR's gating conformational changes involve movements that are analogous to the flip-flop motion seen in ABC transporters (cf. Wang and Linsdell 2012). As more experimental and structural data emerge (Norimatsu et al. 2012), which TMs contribute to the CFTR pore will become clearer.

\section{CFTR Pore Blockers and Vestibules}

CFTR is blocked from the cytosolic side by a diverse group of large organic anions that include a number of commonly used anion channel inhibitors (e.g., glibenclamide) (McDonough et al. 1994; Zhang et al. 2000b; Zhou et al. 2002). The large sizes of some of these blockers and the voltage-dependence of their block indicate the existence of a large intracellular vestibule near the pore entrance (Fig. 5). This vestibule appears to be lined by positively charged residues given that blocker efficacy is reduced by mutating several basic residues in the cytosolic regions of the TMs (e.g., K95, R303, and R352) (Linsdell 2005; St. Aubin and Linsdell 2006; Zhou et al. 2010). As noted

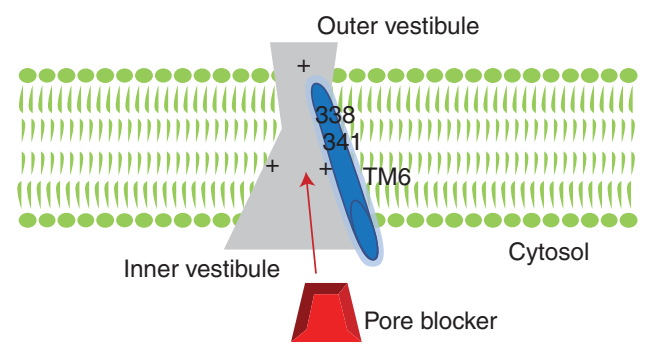

Figure 5. Schematic view of the CFTR permeation pathway. TM6 is indicated as a likely pore-lining helix. The contributions of other TM helices are less clear (see text).

above, the interpretation of such results is complicated by possible effects of mutations on pore structure. In this regard, R352 in TM6 has been proposed to form a salt bridge with D993 in TM9 that stabilizes the open channel conformation (Cui et al. 2008). However, overall, the existing data support the concept of a large, positively charged intracellular vestibule. This charged vestibule should increase the "capture radius" of the pore (Smith et al. 1999) and attract anions to the pore entrance. A similarly charged (but smaller) extracellular vestibule has been proposed to facilitate anion entry from the cell exterior (Smith et al. 2001).

In summary, we have a rudimentary understanding of the pore based on biophysical studies and mutational analyses (Fig. 5). A stringent selectivity filter is not required to explain the CFTR permeability sequence, which is ordered primarily by the hydration energies of the permeant ions. The pore appears to have a narrow region near residues $337-341$ in TM6 that may function as a low grade selectivity filter. Positively charged, asymmetric vestibules at the intracellular and extracellular sides attract anions to the pore entrances to facilitate anion permeation.

\section{CONCLUDING REMARKS}

The unique duality of CFTR as an ATP-gated channel with an intrinsic enzymatic activity that regulates channel closure has fascinated many investigators. Valuable insights and predictions into how CFTR channel gating is regulated by ATP binding, NBD dimerization, and 
ATP hydrolysis have come from recent structural information for its cousins, the ABC exporters. Some features of CFTR channel function are not so easily predicted from the structures of other $\mathrm{ABC}$ transporters, however. These more CFTR-specific issues include (1) how phosphorylation of its unique $\mathrm{R}$ domain regulates gating; (2) how an anion-selective pore is created from the body plan of an $\mathrm{ABC}$ transporter; and (3) the emerging evidence that CFTR gating shares common principles with conventional ligandgated channels. Resolving the latter issues cannot be achieved solely by borrowing structural information from other $\mathrm{ABC}$ transporters. Instead, they await the development of new approaches and tools including high-resolution structures of CFTR per se. As the first drug that targets specifically CFTR gating is in the clinics, mechanistic understanding of CFTR gating will not only shed light on how CFTR potentiators rectify gating defects caused by disease-associated mutations, but also pave the way for the development of complementary strategies for curing cystic fibrosis.

\section{ACKNOWLEDGMENTS}

The authors acknowledge the following grant support from the National Institutes of Health: T.-C.H., R01 HL53445 and R01 DK55835; K.L.K., RO1 HL058341 and RO1 DK056796.

\section{REFERENCES}

Accurso FJ, Rowe SM, Clancy JP, Boyle MP, Dunitz JM Durie PR, Sagel SD, Hornick DB, Konstan MW, Donaldson SH, et al. 2010. Effect of VX-770 in persons with cystic fibrosis and the G551D-CFTR mutation. N Engl J Med 363: 1991-2003.

Aleksandrov L, Aleksandrov AA, Chang XB, Riordan JR. 2002. The first nucleotide binding domain of cystic fibrosis transmembrane conductance regulator is a site of stable nucleotide interaction, whereas the second is a site of rapid turnover. J Biol Chem 277: 15419-15425.

Alexander C, Ivetac A, Liu X, Norimatsu Y, Serrano JR, Landstrom A, Sansom M, Dawson DC. 2009. Cystic fibrosis transmembrane conductance regulator: Using differential reactivity toward channel-permeant and channel-impermeant thiol-reactive probes to test a molecular model for the pore. Biochemistry 48: 10078-10088.

Aller SG, Yu J, Ward A, Weng Y, Chittaboina S, Zhuo R, Harrell PM, Trinh YT, Zhang Q, Urbatsch IL, et al. 2009. Structure of P-glycoprotein reveals a molecular ba- sis for poly-specific drug binding. Science 323: 1718 1722.

Anderson MP, Berger HA, Rich DP, Gregory RJ, Smith AE, Welsh MJ. 1991a. Nucleoside triphosphates are required to open the CFTR chloride channel. Cell 67: 775-784.

Anderson MP, Gregory RJ, Thompson S, Souza DW, Paul S, Mulligan RC, Smith AE, Welsh MJ. 1991b. Demonstration that CFTR is a chloride channel by alteration of its anion selectivity. Science 253: 202-205.

Bai Y, Li M, Hwang TC. 2010. Dual roles of the sixth transmembrane segment of the CFTR chloride channel in gating and permeation. J Gen Physiol 136: 293-309.

Bai Y, Li M, Hwang TC. 2011. Structural basis for the channel function of a degraded ABC transporter, CFTR (ABCC7). J Gen Physiol 138: 495-507.

Baker JMR, Hudson RP, Kanelis V, Choy W-Y, Thibodeau PH, Thomas PJ, Forman-Kay JD. 2007. CFTR regulatory region interacts with NBD1 predominately via multiple transient helices. Nature Struct Mol Biol 14: $738-745$.

Basso C, Vergani P, Nairn AC, Gadsby DC. 2003. Prolonged nonhydrolytic interaction of nucleotide with CFTR's NH2-terminal nucleotide binding domain and its role in channel gating. J Gen Physiol 122: 333-348.

Bear CE, Li CH, Kartner N, Bridges RJ, Jensen TJ, Ramjeesingh M, Riordan JR. 1992. Purification and functional reconstitution of the cystic fibrosis transmembrane conductance regulator (CFTR). Cell 68: 809-818.

Bompadre SG, Ai T, Cho JH, Wang X, Sohma Y, Li M, Hwang TC. 2005a. CFTR gating I: Characterization of the ATP-dependent gating of a phosphorylation-independent CFTR channel (8R-CFTR). J Gen Physiol 125: 361-375.

Bompadre SG, Cho JH, Wang X, Zou X, Sohma Y, Li M, Hwang TC. 2005b. CFTR gating II: Effects of nucleotide binding on the stability of open states. J Gen Physiol 125: 377-394.

Bompadre SG, Sohma Y, Li M, Hwang TC. 2007. G551D and G1349D, two CF-associated mutations in the signature sequences of CFTR, exhibit distinct gating defects. J Gen Physiol 129: 285-298.

Bormann J, Hamill OP, Sakmann B. 1987. Mechanism of anion permeation through channels gated by glycine and $\gamma$-aminobutyric acid in mouse cultured spinal neurons. J Physiol 385: 243-286.

Changeux JP, Edelstein SJ. 2005. Allosteric mechanisms of signal transduction. Science 308: 1424-1428.

Chappe V, Hinkson DA, Howell LD, Evagelidis A, Liao J, Chang X-B, Riordan JR, Hanrahan JW. 2004. Stimulatory and inhibitory protein kinase $\mathrm{C}$ consensus sequences regulate the cystic fibrosis transmembrane conductance regulator. Proc Natl Acad Sci 101: 390-395.

Chen TY, Hwang TC. 2008. CLC-0 and CFTR: Chloride channels evolved from transporters. Physiol Rev 88: 351-387.

Chen J, Lu G, Lin J, Davidson AL, Quiocho FA. 2003. A tweezers-like motion of the ATP-binding cassette dimer in an ABC transport cycle. Mol Cell 12: 651-661.

Cheng SH, Rich DP, Marshall J, Gregory RJ, Welsh MJ, Smith AE. 1991. Phosphorylation of the R domain by 
cAMP-dependent protein kinase regulates the CFTR chloride channel. Cell 66: 1027-1036.

Csanády L, Chan KW, Seto-Young D, Kopsco DC, Nairn AC, Gadsby DC. 2000. Severed channels probe regulation of gating of cystic fibrosis transmembrane conductance regulator by its cytoplasmic domains. J Gen Physiol 116: 477-500.

Csanády L, Seto-Young D, Chan KW, Cenciarelli C, Angel BB, Qin J, McLachlin DT, Krutchinsky AN, Chait BT, Nairn AC, et al. 2005. Preferential phosphorylation of R-domain serine 768 dampens activation of CFTR channels by PKA. J Gen Physiol 125: 171-186.

Csanády L, Nairn AC, Gadsby DC. 2006. Thermodynamics of CFTR channel gating: A spreading conformational change initiates an irreversible gating cycle. J Gen Physiol 128: $523-533$.

Csanády L, Vergani P, Gadsby DC. 2010. Strict coupling between CFTR's catalytic cycle and gating of its $\mathrm{Cl}^{-}$ion pore revealed by distributions of open channel burst durations. Proc Natl Acad Sci 107: 1241-1246.

Cui L, Aleksandrov L, Chang XB, Hou YX, He L, Hegedus T, Gentzsch M, Aleksandrov A, Balch WE, Riordan JR. 2007. Domain interdependence in the biosynthetic assembly of CFTR. J Mol Biol 365: 981-994.

Cui G, Zhang Z-R, O’Brien AR, Song B, McCarty NA. 2008. Mutations at arginine 352 alter the pore architecture of CFTR. J Membr Biol 222: 91-106.

Dawson RJ, Locher KP. 2006. Structure of a bacterial multidrug ABC transporter. Nature 443: 180-185.

Dean M, Annilo T. 2005. Evolution of the ATP-binding cassette $(\mathrm{ABC})$ transporter superfamily in vertebrates. Annu Rev Genomics Hum Genet 6: 123-142.

Diederichs K, Diez J, Greller G, Muller C, Breed J, et al. 2000. Crystal structure of MalK, the ATPase subunit of the trehalose/maltose $\mathrm{ABC}$ transporter of the archaeon Thermococcus litoralis. EMBO J 19: 5951-5961.

Dousmanis AG, Nairn AC, Gadsby DC. 2002. Distinct $\mathrm{Mg}^{2+}$-dependent steps rate limit opening and closing of a single CFTR $\mathrm{Cl}^{-}$channel. J Gen Physiol 119: 545-559.

El Hiani Y, Linsdell P. 2010. Changes in accessibility of cytoplasmic substances to the pore associated with activation of the cystic fibrosis transmembrane conductance regulator chloride channel. J Biol Chem 285: 32126-32140.

Fatehi M, Linsdell P. 2009. Novel residues lining the CFTR chloride channel pore identified by functional modification of introduced cysteines. J Membr Biol 228: 151-164.

Gadsby DC, Vergani P, Csanady L. 2006. The ABC protein turned chloride channel whose failure causes cystic fibrosis. Nature 440: 477-483.

Gaudet R, Wiley DC. 2001. Structure of the ABC ATPase domain of human TAP1, the transporter associated with antigen processing. EMBO J 20: 4964-4972.

Ge N, Muise CN, Gong X, Linsdell P. 2004. Direct comparison of the functional roles played by different transmembrane regions in the cystic fibrosis transmembrane conductance regulator channel pore. J Biol Chem 279: 55283-55289.

Gunderson KL, Kopito RR. 1994. Effects of pyrophosphate and nucleotide analogs suggest a role for ATP hydrolysis in cystic fibrosis transmembrane regulator channel gating. J Biol Chem 269: 19349-19353.
Gunderson KL, Kopito RR. 1995. Conformational states of CFTR associated with channel gating: The role TP binding and hydrolysis. Cell 82: 231-239.

Gutmann DA, Ward A, Urbatsch IL, Chang G, van Veen HW. 2010. Understanding polyspecificity of multidrug ABC transporters: Closing in on the gaps in ABCB1. Trends Biochem Sci 35: 36-42.

He L, Aleksandrov AA, Serohijos AWR, Hegedus T, Aleksandrov LA, Cui L, Dokholyan NV, Riordan JR. 2008. Multiple membrane-cytoplasmic domain contacts in the cystic fibrosis transmembrane conductance regulator (CFTR) mediate regulation of channel gating. J Biol Chem 283: 26383-26390.

Hegedus T, Serohijos AWR, Dokholyan NV, He L, Riordan JR. 2008. Computational studies reveal phosphorylation-dependent changes in the unstructured $\mathrm{R}$ domain of CFTR. J Mol Biol 378: 1052-1063.

Hegedus T, Aleksandrov A, Mengos A, Cui L, Jensen TJ, Riordan JR. 2009. Role of individual $\mathrm{R}$ domain phosphorylation sites in CFTR regulation by protein kinase A. Biochim Biophys Acta 1788: 1341-1349.

Hille B. 1973. Potassium channels in myelinated nerve. Selective permeability to small cations. J Gen Physiol 61: 669-686.

Hung LW, Wang IX, Nikaido K, Liu PQ, Ames GF, Kim SH. 1998. Crystal structure of the ATP-binding subunit of an ABC transporter. Nature 396: 703-707.

Hwang TC, Nagel G, Nairn AC, Gadsby DC. 1994. Regulation of the gating of cystic fibrosis transmembrane conductance regulator $\mathrm{C}_{1}$ channels by phosphorylation and ATP hydrolysis. Proc Natl Acad Sci 91: 4698-4702.

Ishiguro $\mathrm{H}$, Steward MC, Naruse S, Ko SB, Goto H, Case RM, Kondo T, Yamamoto A. 2009. CFTR functions as a bicarbonate channel in pancreatic duct cells. J Gen Physiol 133: 315-326.

Jiang Y, Lee A, Chen J, Ruta V, Cadene M, Chait BT, MacKinnon R. 2003. X-ray structure of a voltage-dependent $\mathrm{K}^{+}$channel. Nature 423: 33-41.

Jordan IK, Kota KC, Cui G, Thompson CH, McCarty NA. 2008. Evolutionary and functional divergence between the cystic fibrosis transmembrane conductance regulator and related ATP-binding cassette transporters. Proc Natl Acad Sci 105: 18865-18870.

Kanelis V, Hudson RP, Thibodeau PH, Thomas PJ, FormanKay JD. 2010. NMR evidence for differential phosphorylation-dependent interactions in WT and $\triangle$ F508 CFTR. EMBO J 29: 263-277.

Karpowich N, Martsinkevich O, Millen L, Yuan YR, Dai PL, MacVey K, Thomas PJ, Hunt JF. 2001. Crystal structures of the MJ1267 ATP binding cassette reveal an induced-fit effect at the ATPase active site of an ABC transporter. Structure 9: 571-586.

Khare D, Oldham ML, Orelle C, Davidson AL, Chen J. 2009. Alternating access in maltose transporter mediated by rigid-body rotations. Mol Cell 33: 528-536.

Kim D, Steward MC. 2009. The role of CFTR in bicarbonate secretion by pancreatic duct and airway epithelia. J Med Invest 56 (suppl): 336-342.

King JD Jr, Fitch AC, Lee JK, McCane JE, Mak DOD, Foskett JK, Hallows KR. 2009. AMP-activated protein 
kinase phosphorylation of the $\mathrm{R}$ domain inhibits PKA stimulation of CFTR. Am J Physiol 297: C94-C101.

Kirk KL, Wang W. 2011. A unified view of cystic fibrosis transmembrane conductance regulator (CFTR) gating: Combining the allosterism of a ligand-gated channel with the enzymatic activity of an ATP-binding cassette (ABC) transporter. J Biol Chem 286: 12813-12819.

Leier I, Jedlitschky G, Buchholz U, Cole SP, Deeley RG Keppler D. 1994. The MRP gene encodes an ATP-dependent export pump for leukotriene C4 and structurally related conjugates. J Biol Chem 269: 27807-27810.

Lewis HA, Buchanan SG, Burley SK, Conners K, Dickey M, Dorwart M, Fowler R, Gao X, Guggino WB, Hendrickson WA, et al. 2004. Structure of nucleotide-binding domain 1 of the cystic fibrosis transmembrane conductance regulator. EMBO J 23: 282-293.

Lewis HA, Zhao X, Wang C, Sauder JM, Rooney I, Noland BW, Lorimer D, Kearins MC, Conners K, Condon B, et al. 2005. Impact of the $\Delta$ F508 mutation in first nucleotide-binding domain of human cystic fibrosis transmembrane conductance regulator on domain folding and structure. J Biol Chem 280: 1346-1353.

Linsdell P. 2005. Location of a common inhibitor binding site in the cytoplasmic vestibule of the cystic fibrosis transmembrane conductance regulator chloride channel pore. J Biol Chem 280: 8945-8950.

Linsdell P. 2006. Mechanism of chloride permeation in the cystic fibrosis transmembrane conductance regulator chloride channel. Exp Physiol 91.1: 123-129.

Linsdell P, Evagelidis A, Hanrahan JW. 2000. Molecular determinants of anion selectivity in the cystic fibrosis transmembrane conductance regulator chloride channel pore. Biophys J 78: 2973-2982.

Liu X, Smith SS, Dawson DC. 2003. CFTR: What's it like inside the pore? J Exp Zool 300A: 69-75.

Loe DW, Almquist KC, Deeley RG, Cole SP. 1996. Multidrug resistance protein (MRP)-mediated transport of leukotriene $\mathrm{C} 4$ and chemotherapeutic agents in membrane vesicles. Demonstration of glutathione-dependent vincristine transport. J Biol Chem 271: 9675-9682.

Mathews CJ, Tabcharani JA, Chang X-B, Jensen TJ, Riordan JR, Hanrahan JW. 1998. Dibasic protein kinase A sites regulate bursting rate and nucleotide sensitivity of the cystic fibrosis transmembrane conductance regulator chloride channel. J Physiol 508.2: 365-377.

McCarty NA, Zhang Z-R. 2001. Identification of a region of strong discrimination in the pore of CFTR. Am J Lung Cell Mol Physiol 281: L852-L867.

McDonough S, Davidson N, Lester HA, McCarty NA. 1994. Novel pore-lining residues in CFTR govern permeation and open-channel block. Neuron 13: 623-634.

Mense M, Vergani P, White DM, Altberg G, Nairn AC, Gadsby DC. 2006. In vivo phosphorylation of CFTR promotes formation of a nucleotide-binding domain heterodimer. EMBO J 25: 4728-4739.

Mornon JP, Lehn P, Callebaut I. 2009. Molecular models of the open and closed states of the whole human CFTR protein. Cell Mol Life Sci 66: 3469-3486.

Nagel G, Hwang TC, Nastiuk KL, Nairn AC, Gadsby DC. 1992. The protein kinase A-regulated cardiac Cl-channel resembles the cystic fibrosis transmembrane conductance regulator. Nature 360: 81-84.

Norimatsu Y, Ivetac AD, Alexander C, Kirkham J, O'Donnell N, Dawson DC, Sansom MS. 2012. CFTR: A molecular model defines the architecture of the anion conduction path and locates a "bottleneck" in the pore. Biochemistry 51: 2199-2212.

Powe AC Jr, Al-Nakkash L, Li M, Hwang TC. 2002. Mutation of Walker-A lysine 464 in cystic fibrosis transmembrane conductance regulator reveals functional interaction between its nucleotide-binding domains. J Physiol 539: 333-346.

Purohit P, Auerbach A. 2009. Unliganded gating of acetylcholine receptor channels. Proc Natl Acad Sci 106: 115120.

Qian F, El Hiani Y, Linsdell P. 2011. Functional arrangement of the 12th transmembrane region in the CFTR chloride channel pore based on functional investigation of a cysteine-less CFTR variant. Pflugers Arch 462: 559-571.

Ramjeesingh M, Li C, Garami E, Huan LJ, Galley K, Wang Y, Bear CE. 1999. Walker mutations reveal loose relationship between catalytic and channel-gating activities of purified CFTR (cystic fibrosis transmembrane conductance regulator). Biochemistry 38: 1463-1468.

Ramsey BW, Davies J, McElvaney NG, Tullis E, Bell SC, Drevinek P, Griese M, McKone EF, Wainwright CE, Konstan MW, et al. 2011. A CFTR potentiator in patients with cystic fibrosis and the G551D mutation. $N$ Engl J Med 365: 1663-1672.

Rees DC, Johnson E, Lewinson O. 2009. ABC transporters: The power to change. Nature Rev 10: 218-227.

Rich DP, Gregory RJ, Anderson MP, Manavalan P, Smith AE, Welsh MJ. 1991. Effect of deleting the R domain on CFTRgenerated chloride channels. Science 253: 205-207.

Rich DP, Berger HA, Cheng SH, Travis SM, Saxena M, Smith AE, Welsh MJ. 1993. Regulation of the cystic fibrosis transmembrane conductance regulator $\mathrm{Cl}^{-}$channel by negative charge in the R domain. J Biol Chem 268: 20259-20267.

Riordan JR, Rommens JM, Kerem B, Alon N, Rozmahel R, Grzelczak Z, Zielenski J, Lok S, Plavsic N, Chou JL, et al. 1989. Identification of the cystic fibrosis gene: Cloning and characterization of complementary DNA. Science 245: 1066-1073.

Rosenberg MF, Mao Q, Holzenburg A, Ford RC, Deeley RG, Cole SP. 2001. The structure of the multidrug resistance protein 1 (MRP1/ABCC1). Crystallization and singleparticle analysis. J Biol Chem 276: 16076-16082.

Schmitt L, Benabdelhak H, Blight MA, Holland IB, Stubbs MT. 2003. Crystal structure of the nucleotidebinding domain of the ABC-transporter haemolysin $\mathrm{B}$ : Identification of a variable region within $\mathrm{ABC}$ helical domains. J Mol Biol 330: 333-342.

Seibert FS, Chang X-B, Aleksandrov AA, Clarke DM, Hanrahan JW, Riordan JR. 1999. Influence of phosphorylation by protein kinase A on CFTR at the cell surface and endoplasmic reticulum. Biochim Biophys Acta 1461: 275-283.

Serohijos AW, Hegedus T, Aleksandrov AA, He L, Cui L, Dokholyan NV, Riordan JR. 2008. Phenylalanine-508 mediates a cytoplasmic-membrane domain contact in the CFTR 3D structure crucial to assembly and channel function. Proc Natl Acad Sci 105: 3256-3261. 
Sheppard DN, Welsh MJ. 1999. Structure and function of the CFTR chloride channel. Physiol Rev 79 (1 Suppl): S23-S45.

Smith SS, Steinle ED, Meyerhoff ME, Dawson DC. 1999. Cystic fibrosis transmembrane conductance regulator: Physical basis for lyotropic anion selectivity patterns. J Gen Physiol 114: 799-818.

Smith SS, Liu X, Zhang Z-R, Sun F, Kriewall TE, McCarty NA, Dawson DC. 2001. CFTR: Covalent and noncovalent modification suggests a role for fixed charges in anion conduction. J Gen Physiol 118: 407-431.

Smith PC, Karpowich N, Millen L, Moody JE, Rosen J, et al 2002. ATP binding to the motor domain from an ABC transporter drives formation of a nucleotide sandwich dimer. Mol Cell 10: 139-149.

St. Aubin CN, Linsdell P. 2006. Positive charges at the intracellular mouth of the pore regulate anion conduction in the CFTR chloride channel. J Gen Physiol 128: 535-545.

Stratford FL, Ramjeesingh M, Cheung JC, Huan LJ, Bear CE. 2007. The Walker B motif of the second nucleotide binding domain (NBD2) of CFTR plays a key role in ATPase activity of the NBD1, NBD2 heterodimer. Biochem J 401: 581-586.

Szollosi A, Vergani P, Csanady L. 2010. Involvement of F1296 and N1303 of CFTR in induced-fit conformational change in response to ATP binding at NBD2. J Gen Physiol 136: 407-423.

Szollosi A, Muallem DR, Csanady L, Vergani P. 2011. Mutant cycles at CFTR's non-canonical ATP-binding site support little interface separation during gating. J Gen Physiol 137: 549-562.

Tsai MF, Shimizu H, Sohma Y, Li M, Hwang TC. 2009. Statedependent modulation of CFTR gating by pyrophosphate. J Gen Physiol 133: 405-419.

Tsai MF, Li M, Hwang TC. 2010. Stable ATP binding mediated by a partial NBD dimer of the CFTR chloride channel. J Gen Physiol 135: 399-414.

Van Goor F, Hadida S, Grootenhuis PD, Burton B, Cao D, Neuberger T, Turnbull A, Singh A, Joubran J, Hazlewood A, et al. 2009. Rescue of CF airway epithelial cell function in vitro by a CFTR potentiator, VX-770. Proc Natl Acad Sci 106: $18825-18830$.

Verdon G, Albers SV, Dijkstra BW, Driessen AJ, Thunnissen AM. 2003. Crystal structures of the ATPase subunit of the glucose $\mathrm{ABC}$ transporter from Sulfolobus solfataricus: Nucleotide-free and nucleotide-bound conformations. J Mol Biol 330: 343-358.

Vergani P, Nairn AC, Gadsby DC. 2003. On the mechanism of MgATP-dependent gating of CFTR Cl- channels. J Gen Physiol 121: 17-36.

Vergani P, Lockless SW, Nairn AC, Gadsby DC. 2005. CFTR channel opening by ATP-driven tight dimerization of its nucleotide-binding domains. Nature 433: 876-880.

Wang W, Linsdell P. 2012. Alternating access to the transmembrane domain of the ATP-binding cassette protein cystic fibrosis transmembrane conductance regulator (ABCC7). J Biol Chem 287: 10156-10165.

Wang F, Zeltwanger S, Hu S, Hwang T-C. 2000. Deletion of phenylalanine 508 causes attenuated phosphorylation- dependent activation of CFTR chloride channels. J Physiol 524: 637-648.

Wang W, Bernard K, Li G, Kirk KL. 2007. Curcumin opens cystic fibrosis transmembrane conductance regulator channels by a novel mechanism that requires neither ATP binding nor dimerization of the nucleotide-binding domains. J Biol Chem 282: 4533-4544.

Wang X, Bompadre SG, Li M, Hwang TC. 2009. Mutations at the signature sequence of CFTR create a $\mathrm{Cd}^{2+}$-gated chloride channel. J Gen Physiol 133: 69-77.

Wang W, Wu J, Bernard K, Li G, Wang G, Bevensee MO, Kirk KL. 2010. ATP-independent CFTR channel gating and allosteric modulation by phosphorylation. Proc Natl Acad Sci 107: 3888-3893.

Ward A, Reyes CL, Yu J, Roth CB, Chang G. 2007. Flexibility in the $\mathrm{ABC}$ transporter MsbA: Alternating access with a twist. Proc Natl Acad Sci 104: 19005-19010.

Wilkinson DJ, Strong TV, Mansoura MK, Wood DL, Smith SS, Collins FS, Dawson DC. 1997. CFTR activation: Additive effects of stimulatory and inhibitory phosphorylation sites in the R domain. Am J Physiol 273: L127-L133.

Wine JJ, Joo NS. 2004. Submucosal glands and airway defense. Proc Am Thorac Soc 1: 47-53.

Yuan YR, Blecker S, Martsinkevich O, Millen L, Thomas PJ, Hunt JF. 2001. The crystal structure of the MJ0796 ATPbinding cassette. Implications for the structural consequences of ATP hydrolysis in the active site of an $\mathrm{ABC}$ transporter. J Biol Chem 276: 32313-32321.

Zeltwanger S, Wang F, Wang GT, Gillis KD, Hwang TC. 1999. Gating of cystic fibrosis transmembrane conductance regulator chloride channels by adenosine triphosphate hydrolysis. Quantitative analysis of a cyclic gating scheme. J Gen Physiol 113: 541-554.

Zhang Z-R, McDonough SI, McCarty NA. 2000a. Interaction between permeation and gating in a putative pore domain mutant in the cystic fibrosis transmembrane conductance regulator. Biophys J 79: 298-313.

Zhang Z-R, Zeltwanger S, McCarty NA. 2000b. Direct comparison of NPPB and DPC as probes of CFTR expressed in Xenopus oocytes. J Membrane Biol 175: 35-52.

Zhang L, Aleksandrov LA, Riordan JR, Ford RC. 2011. Domain location within the cystic fibrosis transmembrane conductance regulator protein investigated by electron microscopy and gold labeling. Biochim Biophys Acta 808: 399-404.

Zhou Z, Hu S, Hwang T-C. 2002. Probing an open CFTR pore with organic anion blockers. J Gen Physiol 120: 647-662.

Zhou Z, Wang X, Li M, Sohma Y, Zou X, Hwang TC. 2005. High affinity ATP/ADP analogues as new tools for studying CFTR gating. J Gen Physiol 569: 447-457.

Zhou Z, Wang X, Liu HY, Zou X, Li M, Hwang TC. 2006. The two ATP binding sites of cystic fibrosis transmembrane conductance regulator (CFTR) play distinct roles in gating kinetics and energetics. J Gen Physiol 128: $413-$ 422.

Zhou J-J, Li M-S, Qi J, Linsdell P. 2010. Regulation of conductance by the number of fixed charges in the intracellular vestibule of the CFTR chloride channel pore. J Gen Physiol 135: 229-245. 


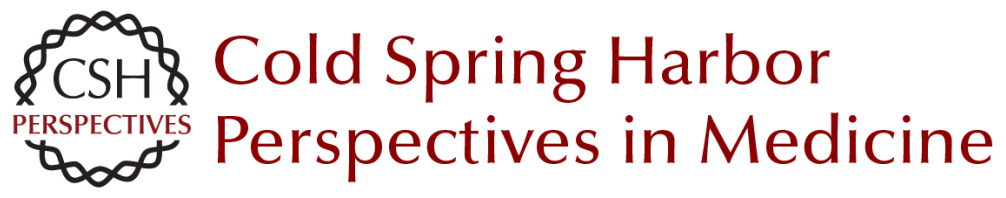

\section{The CFTR Ion Channel: Gating, Regulation, and Anion Permeation}

Tzyh-Chang Hwang and Kevin L. Kirk

Cold Spring Harb Perspect Med 2013; doi: 10.1101/cshperspect.a009498

Subject Collection Cystic Fibrosis

Antibiotic and Anti-Inflammatory Therapies for

Cystic Fibrosis

James F. Chmiel, Michael W. Konstan and J. Stuart Elborn

Structure and Function of the Mucus Clearance System of the Lung

Brenda M. Button and Brian Button

New Pulmonary Therapies Directed at Targets

Other than CFTR

Scott H. Donaldson and Luis Galietta

The Cystic Fibrosis Airway Microbiome

Susan V. Lynch and Kenneth D. Bruce

\section{Cystic Fibrosis Transmembrane Conductance \\ Regulator (ABCC7) Structure \\ John F. Hunt, Chi Wang and Robert C. Ford}

Status of Fluid and Electrolyte Absorption in

Cystic Fibrosis

M.M. Reddy and M. Jackson Stutts

The Influence of Genetics on Cystic Fibrosis

Phenotypes

Michael R. Knowles and Mitchell Drumm

Perspectives on Mucus Properties and Formation

--Lessons from the Biochemical World

Daniel Ambort, Malin E.V. Johansson, Jenny K.

Gustafsson, et al.
The Cystic Fibrosis Intestine

Robert C. De Lisle and Drucy Borowitz

Cystic Fibrosis Transmembrane Regulator

Correctors and Potentiators

Steven M. Rowe and Alan S. Verkman

The Cystic Fibrosis of Exocrine Pancreas

Michael Wilschanski and Ivana Novak

Dynamics Intrinsic to Cystic Fibrosis Transmembrane Conductance Regulator Function and Stability

P. Andrew Chong, Pradeep Kota, Nikolay V.

Dokholyan, et al.

The Cystic Fibrosis Gene: A Molecular Genetic

Perspective

Lap-Chee Tsui and Ruslan Dorfman

The CFTR Ion Channel: Gating, Regulation, and

Anion Permeation

Tzyh-Chang Hwang and Kevin L. Kirk

Assessing the Disease-Liability of Mutations in CFTR

Claude Ferec and Garry R. Cutting

Supramolecular Dynamics of Mucus Pedro Verdugo

For additional articles in this collection, see http://perspectivesinmedicine.cshlp.org/cgi/collection/ 